\title{
Evaluación de variables involucradas en riego superficial para incrementar la eficiencia del uso del agua en la Región Lagunera, Coahuila de Zaragoza, México
}

\author{
Evaluation of variables involved in surface irrigation to increase the efficiency of the use of water in the
} Region Lagunera, Coahuila de Zaragoza, Mexico

\author{
Carlos Miguel Ramos-Cruz ${ }^{1 *}$; Juan Estrada-Ávalos²; Gerardo Delgado-Ramírez²; Emilia Raquel Pérez- \\ Evangelista3; Diego Domínguez-Alemán ${ }^{4}$
}

\begin{abstract}
RESUMEN
En México existe una eficiencia menor a 50\% del agua destinada a la agricultura, por lo que es necesario incrementarla. Esto se puede lograr mejorando el diseño de riego superficial y la aplicación del agua. El objetivo fue evaluar el diseño del riego superficial de los principales cultivos en un módulo de riego en la Región Lagunera, Coahuila de Zaragoza, México. Para esto se midieron longitudes y anchos de melga mediante un Sistema de Información Geográfica; además, se calcularon los gastos de operación. El cultivo de algodón presentó los mayores problemas de diseño de riego: $32.4 \%$ de las parcelas tienen longitudes mayores a $180 \mathrm{~m}$, anchos de $40 \mathrm{~m}$ y gastos unitarios mínimos de $2.0 \mathrm{~L} \mathrm{~s} \mathrm{m-1.} \mathrm{Esto} \mathrm{genera} \mathrm{una} \mathrm{baja} \mathrm{eficiencia} \mathrm{de} \mathrm{aplicación} \mathrm{y,} \mathrm{en}$ consecuencia, menor productividad del agua superficial. Para garantizar una eficiencia de aplicación de $85 \%$ se recomienda considerar las variables involucradas en el diseño de riego.
\end{abstract}

PALABRAS CLAVE

tecnificación del riego, riego superficial, eficiencia de aplicación

\begin{abstract}
In Mexico there is an efficiency of less than $50 \%$ of the water used for agriculture, so it is necessary to increase it. This can be achieved by improving the design of surface irrigation and the application of water. The objective was to evaluate the design of surface irrigation of the main crops in an irrigation module in the Region Lagunera, Coahuila de Zaragoza, Mexico. For this, plot lengths and widths were measured through a Geographical Information System, in addition to operating costs. Cotton crop presented the biggest problems in the irrigation design, $32.4 \%$ of the plots sampled have lengths greater than $180 \mathrm{~m}$, wi$\mathrm{dths}$ of $40 \mathrm{~m}$ and minimum unit costs of $2.0 \mathrm{~L} \mathrm{~s} \mathrm{~m}-1$. This generates a low efficiency of application, consequently, lower surface water productivity. To guarantee an application efficiency of $85 \%$ it is recommended to consider the variables involved in the irrigation design.
\end{abstract}

KEYWORDS

Technification of irrigation, surface irrigation, efficiency of application

\footnotetext{
${ }^{1}$ Campo Experimental, General Terán-INIFAP, km 31 Carretera Montemorelos-China, 67400, General Terán, Nuevo León, México.

${ }^{2}$ Centro Nacional de Investigación Disciplinaria en Relación Agua, Suelo, Planta, Atmósfera (CENID-RASPA) del INIFAP, Canal Sacramento km 6.5, 35140, Gómez Palacios, Durango, México.

${ }^{3}$ Unidad Regional Universitaria de Zonas Áridas, Universidad Autónoma Chapingo, km 38 Carretera Gómez Palacio-Chihuahua, 35230, Bermejillo, Durango, México.

${ }^{4}$ Unidad Laguna, Universidad Autónoma Agraria Antonio Narro, Periférico Raúl López Sánchez y carretera Santa Fe, 27054, Torreón, Coahuila, México.

*Autor para correspondencia: ramos.carlos@inifap.gob.mx
}

Fecha de recepción: 5 de septiembre de 2018

Fecha de aceptación: 9 julio de 2019.

Disponible en línea: 1 de agosto de 2019. 


\section{INTRODUCCIÓN}

En México, se destina $80 \%$ del agua disponible a la agricultura (Martínez et al. 2017), con eficiencia de uso de alrededor de $40 \%$ en los Distritos de Riego (DR) (Catalán et al. 2012) y 57\% en las unidades de riego (Arreguín et al. 2010). Debido a lo anterior, existe potencial para optimizar el agua en el país al incrementar su eficiencia de uso en la agricultura. Para esto, el Gobierno Federal, a través del Programa Nacional Hídrico (PNH) 2014-2018, propuso diversas estrategias que permitan asegurar el agua para riego, la generación de energía, el desarrollo de la industria y el turismo de manera sustentable, al llevar a cabo acciones como la tecnificación del riego, así como el incremento de la eficiencia y la productividad del agua (CNA 2014).

La baja eficiencia del uso del agua a nivel parcelario podría deberse a que en los módulos de riego no se consideran las variables involucradas en el diseño de riego superficial, tales como: longitud y ancho de melgas o camas, nivelación de terreno, gastos unitarios, gasto de entrada a las parcelas y requerimiento hídrico de los cultivos, lo cual está relacionado directamente con la eficiencia de aplicación y las necesidades de riego de los cultivos (Ramos et al. 2018).

El Distrito de Riego 017 (DR-017) se localiza en la Región Lagunera, integrada por los estados de Coahuila y Durango, México. Es administrado por la Comisión Nacional del Agua (CNA) (Pedroza e Hinojosa 2014). Se divide en 20 módulos de riego, de los cuales 17 pertenecen al Río Nazas y el resto al Río Aguanaval, con una superficie dotada total de 223,822 ha y un volumen anual concesionado de $1,024 \mathrm{Mm}^{-3}$ para irrigar 93,409 ha, que dependen del volumen de agua almacenado en la presa Lázaro Cárdenas a inicios del ciclo agrícola (Macías et al. 2007). La distribución del agua se lleva a cabo por medio de una red de 1,266 $\mathrm{km}$ de canales, en beneficio de 37,956 usuarios (García et al. 2010). El sistema de riego empleado para irrigar los cultivos en el DR-017 es superficial por melgas. Sin embargo, este método tiene una baja eficiencia en términos de aplicación, requerimiento y distribución del agua (García-Saldaña et al. 2019). Ramos et al. 2018 señalan que las eficiencias de aplicación promedio en un módulo del DR-017 son de 59.4\%, lo cual indica que los usuarios aplican el doble del agua requerida por los cultivos. Si se consideran adecuadamente las variables de diseño de riego superficial, en la zona bajo estudio se podría incrementar la superficie regable hasta $30 \%$, con el mismo volumen de agua utilizado
(INTA 2015), así como conseguir la aplicación de la lámina de riego requerida por el cultivo y lograr una eficiencia de aplicación alta (Saucedo et al. 2013, Saucedo et al. 2015). El objetivo fue evaluar variables de diseño del riego superficial para los cultivos de algodón, nogal, sorgo forrajero y sorgo escobero en un módulo del Distrito de Riego 017, Región Lagunera, México, para detectar los principales problemas que limitan el óptimo desempeño del riego y proponer estrategias de manejo para incrementar la eficiencia del uso del agua superficial.

\section{MATERIALES Y MÉTODOS}

El estudio se llevó a cabo en un módulo del Distrito de Riego 017 Región Lagunera (DR-017), durante el ciclo agrícola 2013 (periodo marzo-julio). Éste se localiza en el sureste del estado de Coahuila de Zaragoza, entre $25^{\circ} 45^{\prime} 18^{\prime \prime}$ y $25^{\circ} 38^{\prime} 59^{\prime \prime}$ Latitud Norte, $102^{\circ} 46^{\prime} 0.6^{\prime \prime}$ y $103^{\circ} 05^{\prime} 54^{\prime \prime}$ Longitud Oeste a una altitud promedio de 1,096 msnm (figura 1). Cuenta con una red de canales principales de $144 \mathrm{~km}$ y una superficie autorizada para riego, durante el año de evaluación, de 1,862 ha distribuidas en seis secciones de riego. Se evaluaron las secciones 91, 95 y 96, con una superficie total de 1,068 ha, la cual representa $57.3 \%$ de la superficie total del módulo de riego. Los cultivos evaluados se establecieron en 148 parcelas, de las cuales, 74 fueron de algodón, 51 de nogal, 20 de sorgo forrajero y tres de sorgo escobero.

Para la evaluación de las variables del riego superficial en el módulo, se efectuaron las actividades que se describen a continuación.

Integración de un Sistema de Información Geográfica (SIG). Éste se llevó a cabo en el Laboratorio de SIGAgua-Suelo del Centro Nacional de Investigación Disciplinaria en Relación Agua, Suelo, Planta, Atmósfera (CENID RASPA), perteneciente al Instituto Nacional de Investigaciones Forestales, Agrícolas y Pecuarias (INIFAP), con sede en Gómez Palacio, Durango, México, y consistió en digitalizar las coordenadas recolectadas en campo, con el GPS diferencial, de las distintas parcelas evaluadas en polígonos digitales, en formato shapefile, bajo la proyección WGS 84 UTM, para la zona $13 \mathrm{~N}$ (Ramos et al. 2017). Para esto se utilizó Quantum GIS (QGIS), ver. 2.18, desarrollado por la asociación QGIS.ORG, Suiza, software libre y de código abierto (FOSS) (Lakota y Stajnko 2013). 


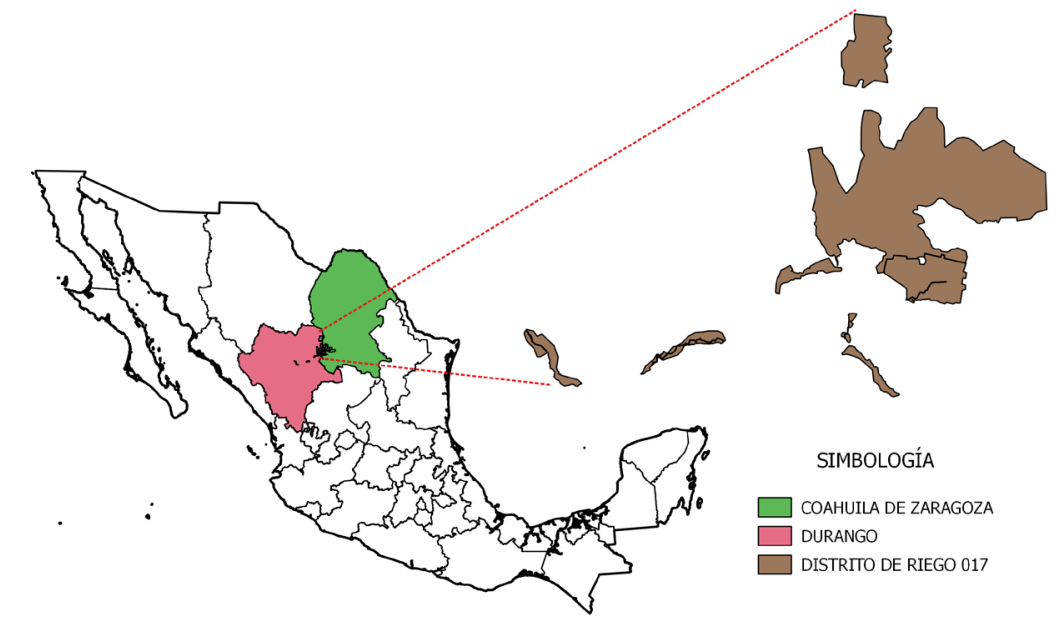

Figura 1. Localización geográfica del Distrito de Riego 017, Región Lagunera, México

Determinación de anchura y longitud de melgas. Para el caso de las longitudes de las melgas se utilizó el SIG generado (Ramos 2016), en el cual se encuentran las longitudes de las parcelas evaluadas. El ancho de melga de la parcela se determinó mediante el uso de imágenes de satélite y la interfaz de Google Earth ${ }^{\circledR}$ Pro Ver. 7.1 (Google, California, USA); posteriormente, estas medidas fueron rectificadas en campo.

Medición del gasto hidráulico. Los aforos se efectuaron a la entrada de cada parcela durante el riego de pre-siembra y tres auxilios para los cultivos de nogal, algodón, sorgo forrajero y sorgo escobero. El gasto hidráulico se calculó por el método de sección velocidad (CNA-IMTA 2001; McPhee et al. 2013). Para determinar el área de la sección de aforo en canales revestidos se midió: base mayor (espejo de agua), tirante hidráulico y base menor (plantilla); asimismo, mediante la fórmula del trapecio (ecuación 1), se calculó el área total de la sección.

$$
A=\frac{B+b}{2} * h
$$

Dónde: $A=$ área de la sección de aforo $\left(\mathrm{m}^{2}\right)$; $B=$ base mayor $(\mathrm{m}) ; b=$ base menor $(\mathrm{m})$ y $h=$ tirante hidráulico $(\mathrm{m})$.
Para el caso de los canales no revestidos, se midió el espejo del agua y se dividió en cuatro dovelas. Posteriormente, se midió el tirante hidráulico de cada dovela; con la ecuación (2) se calculó el área de las dovelas externas, y con la ecuación (3) se calculó el área de las dovelas internas. El área total de la sección de aforo fue la suma de las cuatro dovelas (Ramos et al. 2016).

$$
\begin{aligned}
& A_{1}=\frac{b^{*} h}{2} \\
& A_{2}=\frac{h_{1}+h_{2}}{2} * b
\end{aligned}
$$

Donde: $\mathrm{A}$ = área de la sección de aforo $\left(\mathrm{m}^{2}\right) ; \mathrm{b}$ y $\mathrm{h}$ definidas arriba.

La velocidad se midió con un molinete digital (Goblal Water ${ }^{\circledR}$ modelo FP-111, Goblal Water, Texas, USA), el cual se introdujo al centro de la sección de aforo a una profundidad de $60 \%$ respecto al tirante hidráulico. Con los datos de velocidad y área de los canales se utilizó la ecuación de continuidad para calcular el gasto hidráulico (Pedroza 2017): 


$$
Q=A^{*} V
$$

Donde: $Q=$ Gasto Hidráulico $\left(\mathrm{m}^{3} \mathrm{~s}^{-1}\right) ; A=$ área de la sección de aforo $\left(\mathrm{m}^{2}\right) ; \mathrm{y} V=$ Velocidad del agua $\left(\mathrm{m} \mathrm{s}^{-1}\right)$.

Frentes de riego. Se considera como frente de riego al número de veces en las que se divide el gasto de operación del sistema, es decir, el número de melgas o tendidas que riegan de manera simultánea. Para el caso del presente estudio, esta información se obtuvo en campo, al monitorear el avance de riego en las parcelas evaluadas.

Cálculo de gastos unitarios. Con la medición del gasto parcelario disponible en cada una de las melgas evaluadas de cada parcela y el ancho de éstas, se determinó el gasto unitario, el cual se define dividiendo el gasto parcelario entre el ancho de la melga, ecuación (5) (Delgado et al. 2014).

$$
Q U=\frac{Q}{\mathrm{AM}}
$$

Donde $Q U=$ Gasto unitario $\left(\mathrm{L} \mathrm{s}^{-1} \mathrm{~m}^{-1}\right) ; Q=$ Gasto Hidráulico $\left(\mathrm{m}^{3} \mathrm{~s}^{-1}\right) ; A M=$ Ancho de melga o tendida $(\mathrm{m})$.

\section{RESULTADOS Y DISCUSIÓN}

Con la información recabada en la medición de las longitudes y anchos de melga (cuadro 1), se observa que las longitudes máximas se encuentran en los cultivos de nogal $(256.0 \mathrm{~m})$ y algodón $(247.0 \mathrm{~m})$. Delgado et al. (2014) señalan que las longitudes de las melgas o camas representan un aspecto importante en la mejora del riego superficial, debido a que en longitudes mayores a los $180 \mathrm{~m}$ se dificulta alcanzar una eficiencia mayor a $70 \%$, lo cual se atribuye a la dificultad de controlar la pendiente y el gasto unitario. De acuerdo con lo anterior, $32.4 \%$ de las parcelas de algodón, $29.4 \%$ de nogal y $10 \%$ de sorgo forrajero no cumplen con el criterio antes citado (figura 2). Lo anterior podría deberse a que los usuarios no llevan a cabo un diseño de riego, sino que sólo establecen las melgas conforme al tamaño de sus parcelas (Morales 2004).

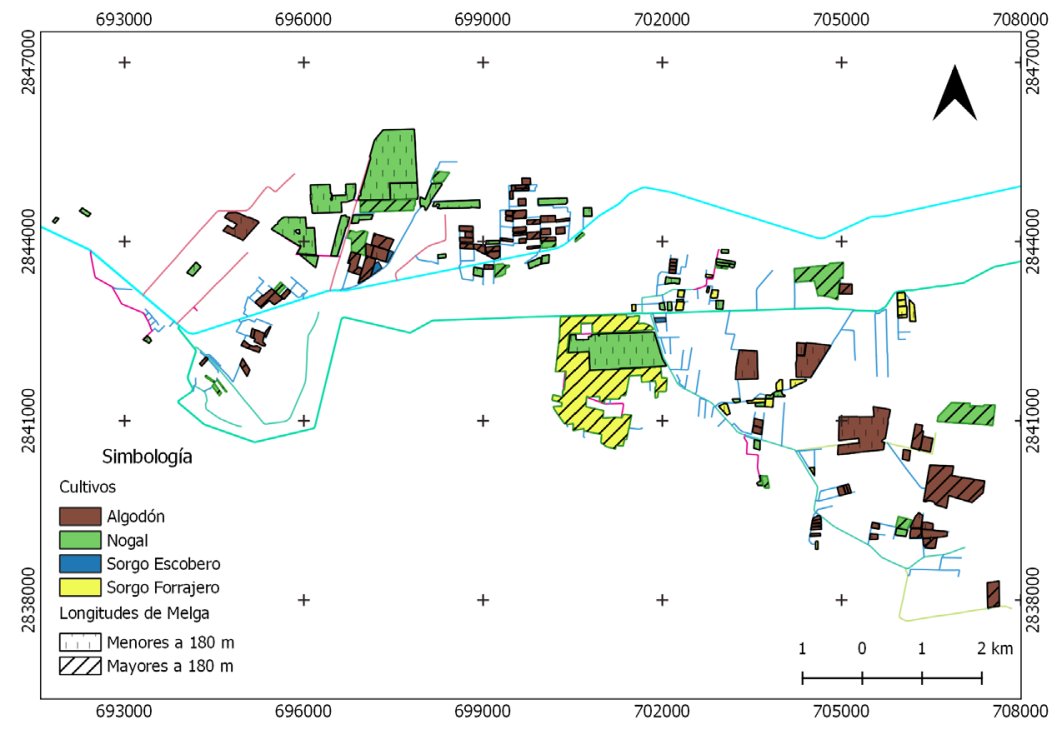

Figura 2. Distribución de las longitudes de melga de los cultivos evaluados en el módulo del Distrito de Riego 017, Región Lagunera, Coahuila de Zaragoza, México 
El ancho de mayor dimensión fue registrado en algodón (40 m) y el de menor en nogal (22 m) (cuadro $1)$; para el caso del nogal, se atribuye principalmente al sistema de plantación, el cual, en promedio, es de 10x10 m (Ramos 2016). Para garantizar una eficiencia de distribución alta, los anchos de las melgas deben estar en concordancia con el gasto unitario (Delgado et al. 2013). En un trabajo efectuado por Delgado et al. (2012), se encontró que al disminuir la longitud de melga de 246 a $123 \mathrm{~m}$, considerar el ancho en función del gasto unitario, así como nivelar el terreno con equipo rayo laser, fue posible incrementar hasta $90 \%$ la eficiencia de aplicación y distribución.

Cuadro 1. Dimensiones de melga de los cultivos presentes en el módulo del Distrito de Riego 017, Región Lagunera, Coahuila de Zaragoza, México

\begin{tabular}{|c|c|c|c|c|c|c|c|}
\hline \multirow[t]{2}{*}{ Cultivo } & \multirow[t]{2}{*}{$\mathbf{n}$} & \multicolumn{3}{|c|}{ Longitud (m-1) } & \multicolumn{3}{|c|}{ Anchura (m-1) } \\
\hline & & Mínima & Máxima & Promedio & Mínima & Máxima & Promedio \\
\hline Nogal & 51 & 50.0 & 256.0 & 140.6 & 18.0 & 22.0 & 20.1 \\
\hline Algodón & 74 & 75.5 & 247.0 & 155.3 & 13.7 & 40.0 & 14.6 \\
\hline Sorgo forrajero & 20 & 64.5 & 201.0 & 121.4 & 17.0 & 21.0 & 19.4 \\
\hline Sorgo escobero & 3 & 95.0 & 180.0 & 135.8 & 19.3 & 20.2 & 19.8 \\
\hline
\end{tabular}

Los gastos obtenidos con los aforos en la bocatoma de las parcelas evaluadas se presentan en el cuadro 2 , donde se observa una variación entre cada uno de los riegos. Los menores gastos se encontraron en el segundo y tercer auxilio (50.3 y $25.7 \mathrm{~L} \mathrm{~s}^{-1}$, respectivamente). De acuerdo con Pedroza e Hinojosa (2014), las variaciones de los gastos se deben a que las tomas no tienen un gasto constante ni en magnitud ni en tiempo, así como a la operación del módulo de riego, el cual hace la distribución con base en los avances de riego.
Para el caso de los frentes de riego (cuadro 2), se encontró que $69 \%$ de los productores de las secciones evaluadas operan con dos frentes de riego (riegan dos melgas o tendidas de manera simultánea). Sin embargo, los usuarios que establecen algodón, son los que llevan a cabo más de dos frentes de riego, disminuyendo el gasto unitario a $1.8 \mathrm{~L} \mathrm{~s} \mathrm{~m}^{-1}$. Lo anterior genera que la lámina de riego aplicada sea más excesiva, el tiempo de riego se incremente y la eficiencia de aplicación disminuya (INTA 2015).

Cuadro 2. Gasto de operación aforados en la bocatoma y frentes de riego observados durante el riego de pre-siembra y tres auxilios de las secciones evaluadas en el módulo del Distrito de Riego 017, Región Lagunera, Coahuila de Zaragoza, México

\begin{tabular}{|c|c|c|c|c|c|c|c|c|}
\hline \multirow[t]{2}{*}{ Riego } & \multirow[t]{2}{*}{ Cultivo } & \multirow[t]{2}{*}{$\mathbf{n}$} & \multicolumn{3}{|c|}{ Gasto de operación ( $\mathrm{L} \mathrm{s}^{-1}$ ) } & \multicolumn{3}{|c|}{ Número de frentes de riego } \\
\hline & & & Mínimo & Máximo & Promedio & Mínimo & Máximo & Promedio \\
\hline \multirow[t]{4}{*}{ Pre-siembra } & Nogal & 51 & 132.4 & 325.7 & 228.8 & 1 & 3 & 1.8 \\
\hline & Algodón & 74 & 123.0 & 361.0 & 201.2 & 2 & 4 & 2.4 \\
\hline & Sorgo forrajero & 20 & 146.0 & 275.6 & 201.0 & 1 & 2 & 1.8 \\
\hline & Sorgo escobero & 3 & 100.1 & 238.4 & 155.9 & 1 & 2 & 1.3 \\
\hline \multirow[t]{4}{*}{ 1er Auxilio } & Nogal & 51 & 120.0 & 360.6 & 214.9 & 1 & 4 & 1.9 \\
\hline & Algodón & 74 & 130.0 & 404.8 & 224.7 & 2 & 4 & 2.9 \\
\hline & Sorgo forrajero & 20 & 145.0 & 334.5 & 182.2 & 1 & 3 & 1.8 \\
\hline & Sorgo escobero & 3 & 46.8 & 204.0 & 151.6 & 1 & 2 & 1.7 \\
\hline
\end{tabular}




\begin{tabular}{lllllllll} 
2do Auxilio & Nogal & 51 & 55.0 & 459.0 & 221.0 & 1 & 4 & 1.9 \\
& Algodón & 74 & 86.8 & 459.6 & 191.3 & 1 & 5 & 2.4 \\
& Sorgo forrajero & 20 & 70.0 & 328.2 & 191.5 & 1 & 3 & 1.9 \\
& Sorgo escobero & 3 & 50.3 & 197.8 & 148.7 & 1 & 2 & 1.7 \\
\multirow{3}{*}{ 3er Auxilio } & Nogal & 51 & 31.6 & 471.0 & 223.1 & 1 & 4 & 1.9 \\
& Algodón & 74 & 25.7 & 604.7 & 183.2 & 1 & 6 & 3 \\
& Sorgo forrajero & 20 & 42.2 & 312.2 & 156.6 & 1 & 1.2 \\
& Sorgo escobero & 3 & 53.0 & 147.7 & 116.1 & 1 & 1.0 & 1.5
\end{tabular}

En el cuadro 3 se muestran los gastos unitarios definidos en la evaluación del ciclo agrícola. Éstos varían entre los distintos riegos; en el caso del algodón, en todos los riegos (pre-siembra, y los tres auxilios), presenta los gastos unitarios promedio más bajos. Esto se debe a que los anchos de melga no son los apropiados en función al gasto parcelario disponible en las secciones evaluadas en el módulo de riego (Ramos 2016). Estos gastos unitarios obtenidos se encuentran por debajo del valor óptimo señalado por Delgado et al. (2014), quienes consignaron que el gasto unitario debe ser 6.0 $\mathrm{L} \mathrm{s} \mathrm{m}^{-1}$, o lo más cercano a este valor en riego superficial, para garantizar una eficiencia potencial mayor a $90 \%$ en la uniformidad de distribución. Gastos unitarios por debajo de $5 \mathrm{~L} \mathrm{~s} \mathrm{~m}^{-1}$ tienden a infiltrar mayor cantidad de agua en la cabecera de la melga, lo cual disminuye la uniformidad de distribución a lo largo de la misma. En contraste, con gastos unitarios mayores a los $7 \mathrm{~L} \mathrm{~s} \mathrm{~m}^{-1}$, se tienden a infiltrar mayor cantidad de agua hacia el recibidor de la melga, provocando el efecto contrario, así como favorecer la erosión del suelo (Delgado et al. 2013). Otros aspectos que deben ser considerados para incrementar la eficiencia de aplicación y distribución del riego superficial son: efectuar análisis de suelo para conocer los parámetros físicos (capacidad de retención de humedad, textura, densidad aparente, etc.); medición y monitoreo de los gastos parcelarios aplicados en las parcelas, y capacitación continua a usuarios, gerentes, canaleros y regadores del módulo de riego.

Cuadro 3. Gastos unitarios observados durante el riego de pre-siembra y tres auxilios de las secciones evaluadas en Distrito de Riego 017, Región Lagunera, Coahuila de Zaragoza, México

\begin{tabular}{|c|c|c|c|c|c|}
\hline Riego & $\mathbf{n}$ & Cultivo & Mínimo $\left(\mathrm{L} \mathrm{s} \mathrm{m}^{-1}\right)$ & Máximo ( $\left.\mathrm{L} \mathrm{s} \mathrm{m}^{-1}\right)$ & Promedio ( $\left.\mathrm{L} \mathrm{s} \mathrm{m}^{-1}\right)$ \\
\hline \multirow[t]{4}{*}{ Pre-siembra } & 51 & Nogal & 4.1 & 8.0 & 6.4 \\
\hline & 74 & Algodón & 3.6 & 7.5 & 5.7 \\
\hline & 20 & Sorgo forrajero & 4.1 & 7.9 & 6.2 \\
\hline & 3 & Sorgo escobero & 5.0 & 6.5 & 5.9 \\
\hline \multirow{4}{*}{$\begin{array}{l}\text { 1er } \\
\text { Auxilio }\end{array}$} & 51 & Nogal & 4.2 & 7.8 & 6.1 \\
\hline & 74 & Algodón & 3.2 & 7.0 & 5.4 \\
\hline & 20 & Sorgo forrajero & 4.0 & 7.8 & 5.7 \\
\hline & 3 & Sorgo escobero & 2.3 & 5.3 & 4.2 \\
\hline \multirow[t]{4}{*}{ 2do Auxilio } & 51 & Nogal & 2.8 & 7.9 & 5.8 \\
\hline & 74 & Algodón & 3.6 & 7.4 & 5.6 \\
\hline & 20 & Sorgo forrajero & 3.6 & 7.5 & 5.2 \\
\hline & 3 & Sorgo escobero & 2.5 & 5.1 & 4.2 \\
\hline \multirow{4}{*}{$\begin{array}{l}\text { 3er } \\
\text { Auxilio }\end{array}$} & 51 & Nogal & 1.6 & 7.8 & 6.0 \\
\hline & 74 & Algodón & 1.8 & 7.2 & 5.5 \\
\hline & 20 & Sorgo forrajero & 2.2 & 7.8 & 5.6 \\
\hline & 3 & Sorgo escobero & 2.6 & 7.7 & 5.9 \\
\hline
\end{tabular}




\section{CONCLUSIONES}

Se concluye que los usuarios del módulo definen las variables de riego (longitud, ancho y pendiente longitudinal) con base en su experiencia. Esto ocasiona bajas eficiencias en la operación del riego (distribución, conducción y aplicación del agua a las parcelas), en particular, para garantizar una eficiencia de aplicación de por lo menos $85 \%$ en el riego superficial del módulo. Se recomienda que, al inicio del establecimiento de los cultivos, el diseño de riego cubra las siguientes características: longitudes de melga no mayores a $180 \mathrm{~m}$; ancho de melga en relación con el gasto unitario; gastos unitarios de 6.0 L s m${ }^{-1}$, así como la nivelación del terreno con una pendiente longitudinal en relación a la textura del suelo, además de considerar los requerimientos hídricos de los cultivos.

\section{LiTERATURA CITADA}

Arreguín F, Alcocer V, Marengo H, Cervantes C, Albornoz P, Salinas MG. 2010. Los retos del agua. En: El agua en México: Cauces y encauces (B. Jiménez, M. L. Torregrosa y L. Aboites, eds.), pp. 51-79. Comisión Nacional del Agua, México.

Catalán E, Villa MM, Inzunza M, Román A, González J. 2012. Cálculo de demandas de agua y programación del riego de cultivos en Coahuila. AGROFAZ 12 (3): 123-131.

[CNA] Comisión Nacional del Agua. 2014. Plan Nacional de Desarrollo 2014-2018. Programa Nacional Hídrico. Coyoacán, México.

[CNA-IMTA] Comisión Nacional del Agua-Instituto Mexicano de Tecnología del Agua 2001. Aforos de descargas. Serie autodidáctica de medición de la calidad del agua. Jiutepec, Morelos, México.

Delgado G, Estrada J, Rivera M, Catalán E, Esquivel G. 2014. Evaluación y diseño del riego por melgas mediante un modelo de simulasión. AGROFAZ 14 (2): 45-51.

Delgado G, Anaya A, Estrada J, Trucíos R, Catalán E. 2012. Evaluación y diseño del riego superficial en melgas mediante un modelo de simulación. Memoria de la XXIV Semana Internacional de Agronomía FAZ-UJED. Gómez Palacios, Durango, México.

Delgado G, Estrada J, Trucíos R, Rivera M, Catalán E. 2013. Metodología para la evaluación de la eficiencia global del riego en sistemas tipo válvulas alfalferas: Caso Región Lagunera. Revista Chapingo Serie Zonas Áridas 12(1): 3-6.
García-Saldaña A, Landeros-Sánchez C, Castañeda-Chávez MR, Martínez-Dávila JP, Pérez-Vázquez A, Carrillo-Ávila E. 2019. Fertirrigation with low-pressure multi-gate irrigation systems in sugarcane agroecosystems: A review. Pedosphere 29: 1-11. https://doi.org/10.1016/ S1002-0160(18)60053-0

García MY, Sánchez I, García G, Moreno L, Trejo R, Hernández MA. 2010. Evaluación de la eficiencia de riego en el Módulo IV del Distrito de Riego 017 Comarca Lagunera, México. Revista Chapingo Serie Zonas Áridas 9(1): 99-106.

[INTA] Instituto Nacional de Tecnología Agropecuaria. 2015. Manual de capacitación: riego superficial. Ciudad Autónoma de Buenos Aires, Argentina.

Lakota M, Stajnko D. 2013. Using of GIS tools for analysis of organic waste management in Slovenia region Pomurje. 6th International Conference on Information and Communication Technologies in Agriculture, Food and Environment (HAICTA 2013). Corfu Island, Greece.

Macias H, Sánchez I, Catalán EA. 2007. Sistema soporte de decisiones para el manejo integral del agua en el DR 017, Coahuila y Durango, México. Revista Chapingo Serie Zonas Áridas 6(1): 77-89.

Martínez E, Espitia E, Villaseñor H, Hortelano R, Muñis E, Zamudio A. 2017. Calidad industrial del trigo harinero en función del número de riegos. Revista Mexicana de Ciencias Agrícolas 8 (7); 1497-1508.

McPhee J, Fernández S, Richard J. 2013. Guía de aforos en canales abiertos y estimación de tasas de infiltración. Facultad de Ciencias Físicas y Matemáticas Universidad de Chile. Santiago, Chile.

Morales J. 2004. Sustentabilidad de los sistemas de riego en el norte de México: caso Región Lagunera. Tesis de Doctorado. Universidad Juárez del Estado de Durango. Venecia, Durango.

Pedroza E. 2017. El molinete, importancia, fundamentos y buenas prácticas. Instituto Mexicano de Tecnología del Agua. Jiutepec, México.

Pedroza E, Hinojosa GA. 2014. Manejo y distribución del agua en distritos de riego: breve introducción didáctica. Instituto Mexicano de Tecnología del Agua, Jiutepec, Morelos. México.

Ramos CM. 2016. Productividad del agua en un módulo del distrito 017 Región Lagunera. Tesis de Maestría. Universidad Juárez del Estado de Durango. Venecia, México.

Ramos CM, Estrada J, Delgado G, Miguel, Domínguez, D. 2018. Estimación de la eficiencia de riego superficial parcelario en un módulo del distrito 017 Región Lagunera. Revista Chapingo Serie Zonas Áridas 17(2): 21-30. https://doi.org/10.5154/r.rchsza.2018.04.011

Ramos CM, Delgado G, Estrada J, Orona I, Miguel E. 2016. Productividad del agua superficial de riego en un módulo del Distrito 017 Región Lagunera. Memoria de 
la XXVIII Semana Internacional de Agronomía FAZUJED. Gómez Palacios, Durango, México.

Ramos CM, Estrada J, Delgado G, Miguel E, Servín, A. 2017. Metodología para la integración de un SIG a nivel módulo de riego mediante Software de código libre y topografía de precisión. Memoria de la XXIX Semana Internacional de Agronomía FAZ-UJED. Gómez Palacios, Durango, México.

Saucedo H, Zavala M, Fuentes C, Castanedo V. 2013. Gasto óptimo en el riego por melgas. Tecnología y Ciencias del Agua 4(3): 135-148.

Saucedo H, Zavala M, Fuentes C. (2015). Diseño de riego por melgas empleando las ecuaciones de Saint-Venant, y Green y Ampt. Tecnología y Ciencias del Agua 6(5): 103-112. 